\title{
Manejo del Prolapso Genital
}

\author{
Dr. Germán Uriza Gutiérrez \\ Autor \\ Profesor Asistente de \\ Ginecología y Obstetricia \\ Pontificia Universidad Javeriana
}

\author{
Dr. Jorge Medina Murillo \\ Coautor \\ Profesor Titular de \\ Ginecología y Obstetricia \\ Pontificia Universidad Javeriana
}

\section{INTRODUCCION}

El prolapso genital se conoce en la historia de la medicina desde hace 4.000 años, encontrándose el primer informe sobre este padecimiento en los papiros de Ebers 9 .

El avance en su tratamiento fue muy lento, agravado por el estancamiento y aun atraso que significó para todos los campos de la ciencia la Edad Media. Sólo con el advenimiento de la cirugía se llegó al método efectivo de tratar el prolapso genital; este avance se inició en el siglo XIX con el desarrollo de las técnicas quirúrgicas, anestesia y los medios de sutura.

Aún en nuestros días están vigentes técnicas descritas hace muchos años, entre las cuales se pueden nombrar la colpocleisis de Le Fort, descrita en 1877; la operación de Mánchester, descrita por Donald en 1888 y modificada por Fotherhill en 1908; la operación de Kelly, descrita en 1912, y la histerectomía vaginal tipo Heney, descrita en el año de 19349.

El prolapso genital es un término genérico que agrupa la procidencia de varios órganos, a saber: la vagina, la uretra, la vejiga, el útero y el recto. La dolencia se clasifica en grados por separado, encontrándose con gran frecuencia en combinación diferentes grados de prolapso de las distintas estructuras. Es de anotar que hay autores que utilizan el término "prolapso" únicamente para el histerocele 1, 12 .

En este estudio utilizamos la clasiticación en 3 grados: el Grado I, cuando el órgano prolapsado se encuentra en el tercio inferior de la vagina; el Grado II, cuando se encuentra a nivel del introito; y el Grado III, cuando sale de la vulva 12

Es tan común esta afección que casi toda mujer que ha tenido hijos tiene, en mayor o menor grado, prolapso genital, no queriendo decir con esto que todas ellas requieran tratamiento quirúrgico 11 .

En el departamento de Ginecología y Obstetricia del Hospital San Ignacio, el prolapso genital constituye una de las causas más frecuentes de consulta. $\mathrm{Pa}$ ra el manejo de estos casos hemos unificado nuestros criterios en los últimos diez años, siendo el objeto de este trabajo el análisis de los casos de prolapso genital.

En Colombia se han hecho trabajos similares al nuestro en el Hospital San Vicente de Paúl en Medellín 1 y en el Hospital San Juan de Dios de Bogotá; sin embargo, hemos considerado importante resımir nuestros resultados con el fin de poder fijar unas pautas en el manejo del prolapso genital, de acuerdo con los últimos adelantos técnicos y con nuestra propia experiencia. 


\section{Material y métodos}

Se revisaron todas las historias de las pacientes intervenidas para corrección del prolapso genital, en un período de 6 años, comprendido entre el $1^{\circ}$ de enero de 1966 y el 31 de diciembre de 1971.

Se recolectaron 432 historias que constituyen el universo de esta investigación, las cuales corresponden al $31.4 \%$ del total de la cirugía ginecoló. gica practicada en este período, exceptuando los legrados uterinos. Los datos se recolectaron en formularios elaborados para tal fin (Anexo 1) y usando un instructivo para la recolección correcta de la información (Anexo 2). Los datos recolectados fueron limpiados, codificados y pasados a tarjetas para su procesamiento por computador. Finalmente, los datos fueron analizados encontrando los siguientes resultados:

1. Edad: La distribución por edades, varía desde los 19 hasta los 73 años. El porcentaje mayor se encuentra entre los 40 y los 59 años y representa el $59.7 \%$ del total (Tabla $\mathrm{N} \div 1$ ).

TABLA N: 1

Distribución por edades

\begin{tabular}{lrc}
\hline Edad-años & No casos & Porcentaje \\
\hline-20 & 2 & 0.5 \\
$20-29$ & 26 & 6.0 \\
$30-39$ & 75 & 17.4 \\
$40-49$ & 150 & 34.7 \\
$50-59$ & 108 & 25.0 \\
$60-69$ & 57 & 13.2 \\
$70-79$ & 13 & 3.0 \\
Sin dato & 1 & 0.2 \\
\hline Total & 432 & 100.0 \\
\hline
\end{tabular}

2. Paridad: Es de anotar que se encontró prolapso en 7 pacientes nulíparas, siendo 16 la mayor paridad. EI
$64.4 \%$ de las pacientes fueron grandes multíparas o sea con más de 5 partos (Tabla $\mathrm{N}^{\circ}$ 2).

\section{TABLA N $\div 2$}

Paridad

\begin{tabular}{lcr}
\hline Paridad & N: casos & Porcentaje \\
\hline Nulíparas & 7 & 1.6 \\
$1-4$ & 146 & 33.8 \\
$5-7$ & 126 & 29.2 \\
Más de 7 & 152 & 35.2 \\
Sin dato & 1 & 0.2 \\
\hline Total & 432 & 100.0 \\
\hline
\end{tabular}

3. Citología: Se practicó dicho examen en 388 casos $(89.2 \%)$ con los siguientes resultados: PAP I, 213 (54.90 $\%)$; PAP II, $160(41.20 \%)$; PAP III, 10 $(2.6 \%)$; PAP IV, $3(1.0 \%)$; y PAP V. 2 $(0.5 \%)$. De las 15 citologías positivas, correspondieron a $\mathrm{Ca}$ in situ, (las 3 PAP IV, las 2 PAP $V$ y una de las PAP III). No hubo ningún caso de $\mathrm{Ca}$ invasivo del cervix. Se encontraron 5 displasias, correspondiendo todas a citología PAP III, y un adenocarcinoma de endometrio cuya citología fue PAP II (Tabla № 3).

\section{TABLA N:3}

Citologías

\begin{tabular}{lrc}
\hline Citología & No casos & Porcentaje \\
\hline PAP I & 213 & 54.9 \\
PAP II & 160 & 41.2 \\
PAP III & 10 & 2.6 \\
PAP IV & 3 & 1.0 \\
PAP V & 2 & 0.5 \\
\hline Total & 388 & 100.0 \\
\hline
\end{tabular}

4. Incontinencia urinaria y patología asociada: $\mathrm{La}$ incontinencia urinaria de esfuerzo es un sintoma que acom- 
paña frecuentemente al prolapso genital y se define como el escape involuntario de orina al hacer esfuerzos cuando la presión intravesical es mayor que la presión uretral máxima, situación que se presenta con la relajación de las estructuras de sostén de la uretra y la vejiga sin que exista actividad del de. trusor 16 .

Los datos se obtuvieron por análisis de los síntomas referidos por la paciente y confirmados objetivamente al hacer la prueba del esfuerzo con la vejiga llena 14 . En nuestro estudio se encontró este síntoma en 207 pacientes, que constituyen el $47.9 \%$ del total.

La otra patología asociada más frecuente fue la hipertensión arterial en 183 casos $(42.4 \%)$. Las afecciones ginecológicas asociadas más frecuentes fueron: leiomiomatosis, $56(13 \%)$; adenomiosis, $46(11 \%)$; hiperplasia endometrial, $26(6 \%)$ (16 adenomatosas, 6 quísticas y 4 simples); poliposis endometrial, $22(5.1 \%)$; quistes de ovario, $8(1.9 \%)$ (6 simples, uno indeterminado y un cistadenoma pseudomucionoso).

Las afecciones ginecológicas malignas fueron $7(1.6 \%)$ (6 carcinomas in situ y un adenocarcinoma de endometrio) (Tabla $N^{\circ} 4$ ).
TABLA N $N 4$

\begin{tabular}{lrr}
\hline Patología asociada & \multicolumn{1}{c}{$\mathrm{N}^{\circ}$} & $\%$ \\
\hline Incontinencia urinaria & 207 & 47.9 \\
Hipertensión arterial & 183 & 42.4 \\
Leiomiomatosis & 56 & 13.0 \\
Adenomiosis & 46 & 11.0 \\
Hiperplasia endometrial & 26 & 6.0 \\
Pólipos endometriales & 22 & 5.1 \\
Quistes de ovario & 8 & 1.9 \\
CA in situ & 6 & 1.4 \\
Adenocarcinoma & & \\
endometrial & 1 & 0.2 \\
\hline
\end{tabular}

5. Tiempo de evolución del prolapso: El tiempo entre la aparición de los síntomas y el tratamiento, en su mayoría pasó de un año, encontrándose el mayor número de pacientes en el grupo de uno a cinco años, con 187 casos $(43.3 \%)$.

6. Grado de prolapso y desgarro perineal: En la tabla $N^{\circ}$ : 5 se muestra la distribución de los grados de prolapso en las diferentes estructuras, que fueron objeto de corrección quirúrgica.

7. Edad y grado de histerocele: Al analizar la relación que puede existir entre la edad y el grado de descenso uterino (histerocele), vemos en el gráfico $N^{\circ} 1$ como el grado de prolapso

TABLA № 5

Grados de prolapso y estructura prolapsada

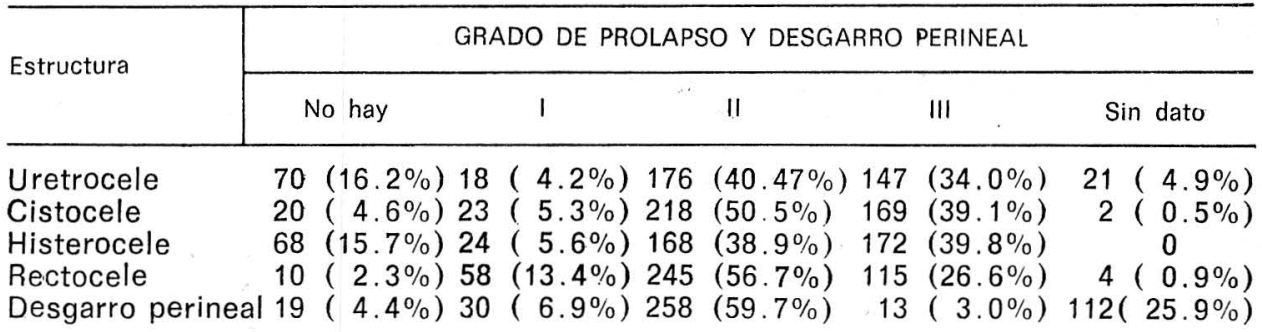


GRAFICO No. 1

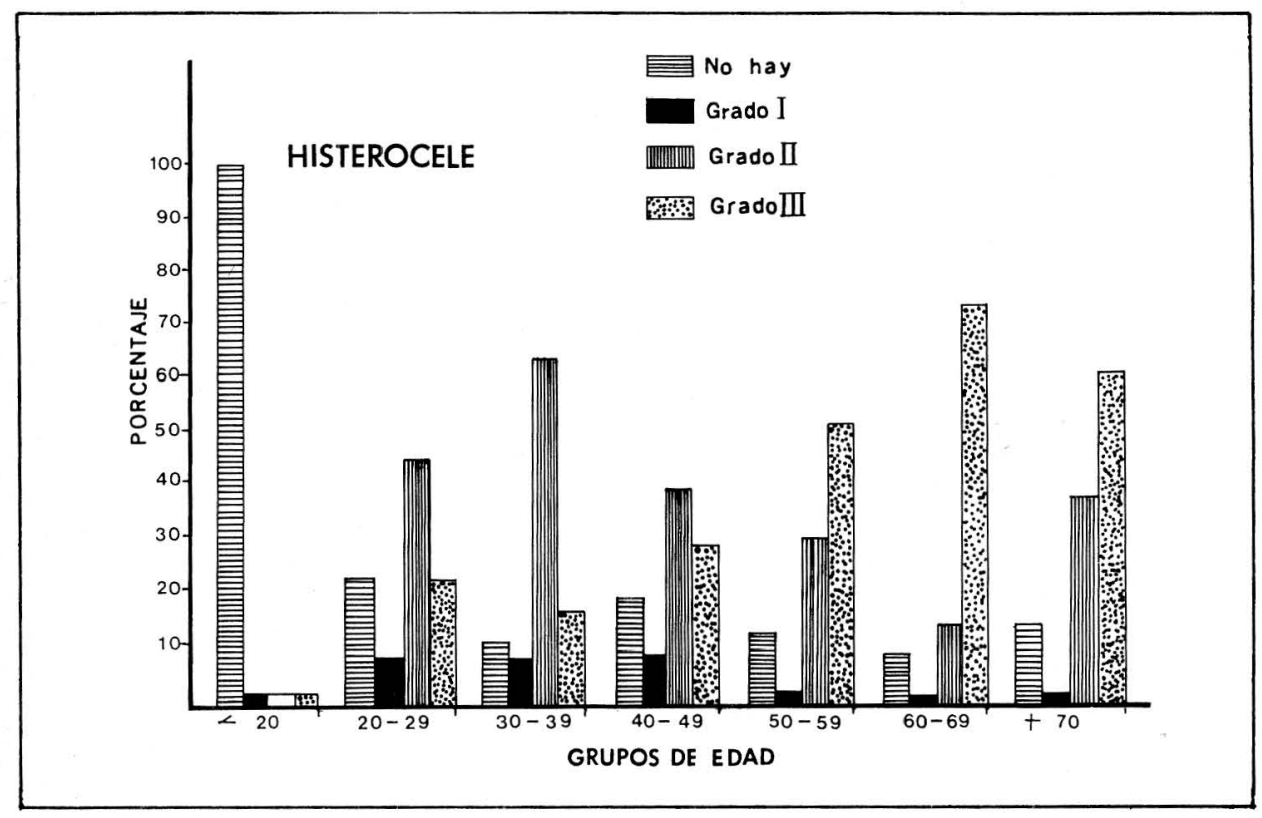

uterino sigue una secuencia en la vida de la mujer y a medida que aumenta la edad hay un predominio de histerocele Grado III, encontrándose que en el grupo de 50 a 59 el histerocele III corresponde al $52.8 \%$, mientras que en el de 60 a 69 años corresponde al $75.4 \%$.

El histerocele Grado II predominó en el grupo de 30 a 39 años, $(64 \%)$ con una tendencia descendente a medida que avanza la edad, encontrándose en un $15.8 \%$ en el grupo de 60 a 69 años. No hubo histerocele en ninguna mujer menor de 20 años.

8. Histerocele y paridad: En el gráfico 2 comparados el grado de histerocele con la paridad vemos que este es muy raro en la paciente nulípara $(7 \%)$, pero una vez que la mujer ha tenido hijos, el grado de histerocele no aumenta proporcionalmente al número de hijos, como sí lo hace proporcionalmente con la edad.
9. Prolapso de cúpula: Se encontraron 13 prolapsos de cúpula, que corres ponden al $3 \%, 6$ casos después de histerectomía vaginal y 7 casos después de histerectomía abdominal. Puede verse que el tipo de histerectomía no incidió en favor de la aparición del prolapso de cúpula. 4 casos se corrigieron vía vaginal; 7 , con operación de Fletcher, y 2 con fijación presacra y uretrocistopexia 15.

10. Tipo de tratamiento: Como se puede apreciar en la tabla $N$ ㅇ 6 , el mayor tipo de intervención practicado fue la histerectomía vaginal, con 325 casos $(75.2 \%)$. El resto de las intervenciones constituyen el $24.8 \%$ del total.

11. Tipo de tratamiento y grupo de edad: Si comparamos en un gráfico el tipo de tratamiento relacionándolo con el grupo de edad, podemos ver dos hechos fundamentales: 
GRAFICO No. 2



a) El predominio de las histerectomías vaginales sobre los otros tipos de tratamiento, encontrando sólo mayoría de tratamientos conservadores en las menores de 30 años. b) Cómo determinadas cirugías tienen su indicación sólo en determinados grupos de edad, cómo es el Mánchester en pacientes entre los 20 y los 40 años. $y$ el Le Fort en pacientes mayores de 60 años. Gráfico № 3.

TABLA № 6

Tipo de tratamiento

\begin{tabular}{lrr}
\hline \multicolumn{1}{c}{ Tratamiento } & Casos & Porcentaje \\
\hline Histerectomía vaginal & 325 & 75.3 \\
Colpoperineorrafia & 33 & 7.6 \\
Mánchester & 19 & 4.4 \\
Combinado con laparotomía & 19 & 4.4 \\
Perineorrafia & 10 & 2.3 \\
Colporrafia anterior & 10 & 2.3 \\
Flechter & 7 & 1.6 \\
Le Fort & 4 & 0.9 \\
Otros & 5 & 1.2 \\
\hline Total & 432 & 1.00 .0 \\
\hline
\end{tabular}


(En el gráfico, con el fin de simplicar un poco, se han agrupado en una sola línea la colporrafia anterior, la perineorrafia y la colpoperineorrafia, tomando por separado los otros tipos de tratamiento).

\section{Tipo de tratamiento y grado de pro-} lapso: Tomamos como representativo del grado de prolapso, al histerocele. En la tabla $\mathrm{N}: 7$ podemos observar varias cosas: Primero: el predominio de los prolapsos Grados II $(38.9 \%)$ y III $(39.8 \%)$. Segundo: el predominio absoluto de la histerectomía vaginal sobre los otros tipos de tratamiento, cosa que ya habíamos visto en el gráfico № 3 . Tercero: la corrección de celes como tratamiento único, se practicó en su mayoría cuando no había histerocele; en todos los casos de colporrafia anterior y perineorrafia, no había histerocele; en la colpoperineorrafia, en el $75.8 \%$ no habia histerocele y solamente en 8 casos $(29.2 \%)$, este existía pero en Grado I. Cuarto: no se requiere en forma absoluta la presencia de his- rerocele en Grados II o III para practicar la histerectomía vaginal, ya que ésta se practicó en 15 casos de histerocele I y en un caso en el cual no había histerocele.

13. Complicaciones: Se encuentran descritas en la tabla $\mathrm{N}$ : 8. Las hemos dividido en: a) Complicaciones operatorias, las que se presentan durante la cirugía; b) Complicaciones postoperatorias inmediatas, las que se presentan durante la permanencia postoperatoria en el hospital; y c) Complicaciones postoperatorias tardías, las que se encontraron en el control postoperatorio, después de haber salido del hospital.

a) Operatorias: Ninguna muerte operatoria. La complicación más frecuente fue la transfusión en 238 casos $(55.1 \%)$; de éstos, 15 necesitaron más de 1.000 cc de sangre, y 128 necesitaron solamente $500 \mathrm{cc}$. El resto de complicaciones operatorias tuvo un porcentaje mínimo.

GRAFICO No. 3

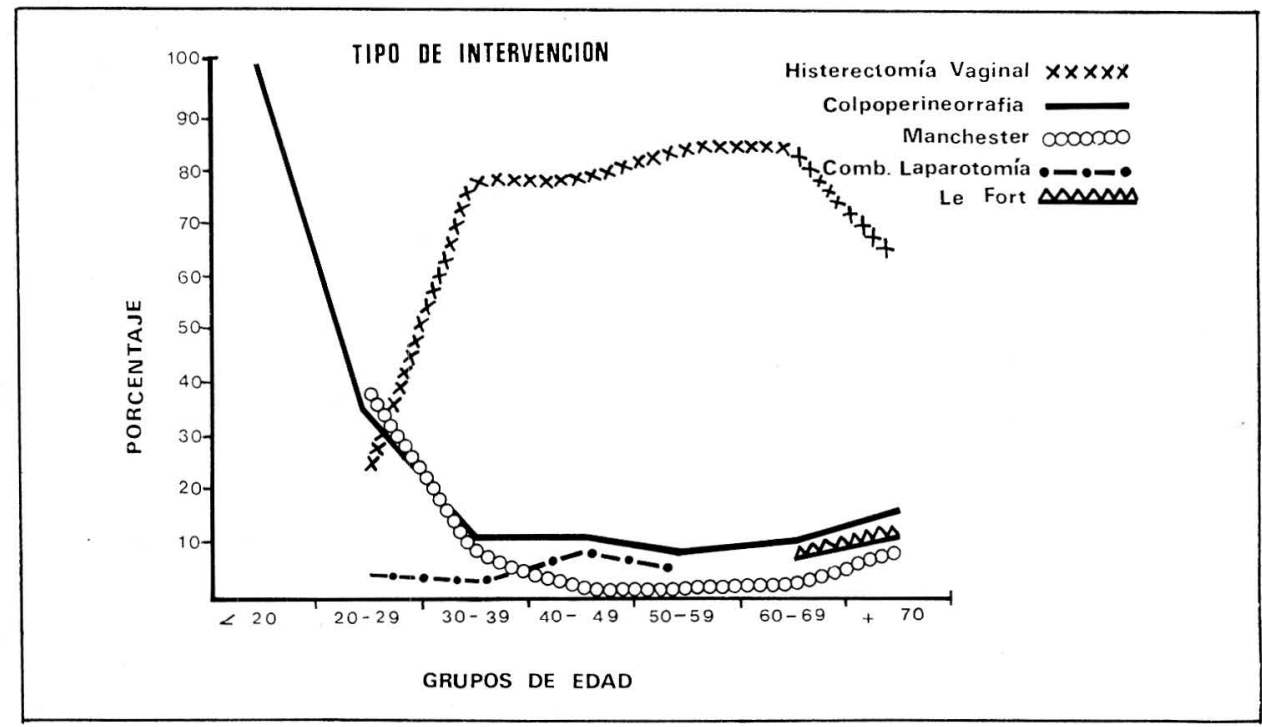


TABLA N: 7

Tipo de tratamiento y grado de prolapso

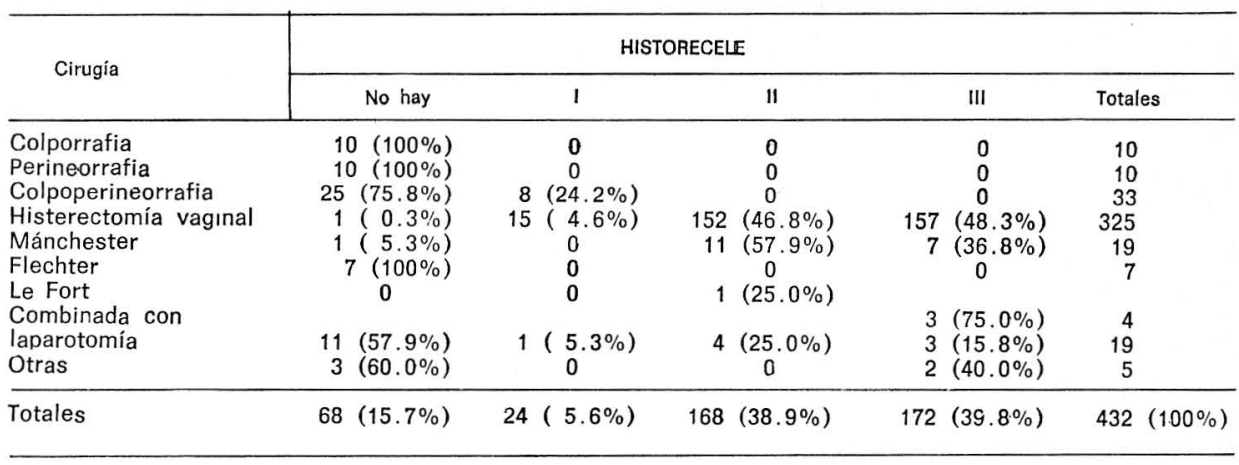

TABLA № 8

Complicaciones

\begin{tabular}{|c|c|c|c|}
\hline Complicación & Tipo & No & $\%$ \\
\hline Operatoria & $\begin{array}{l}\text { Transfusión } \\
\text { Lesión vejiga } \\
\text { Lesión recto } \\
\text { Shock } \\
\text { Lesión uretra } \\
\text { Ligadura uréter } \\
\text { Laparotomía } \\
\text { Muerte }\end{array}$ & $\begin{array}{r}238 \\
7 \\
7 \\
6 \\
3 \\
2 \\
1 \\
0\end{array}$ & $\begin{array}{l}55.1 \\
1.6 \\
1.6 \\
1.4 \\
0.7 \\
0.5 \\
0.2 \\
0\end{array}$ \\
\hline Postoperatoria inmediata & $\begin{array}{l}\text { Retención urinaria } \\
\text { Fiebre } \\
\text { Infección urinaria } \\
\text { Absceso de cúpula } \\
\text { Infección suturas } \\
\text { Hemorragia vaginal } \\
\text { Hemoperitoneo } \\
\text { Peritonitis } \\
\text { Muerte } \\
\text { Obstrucción intestinal }\end{array}$ & $\begin{array}{r}181 \\
147 \\
89 \\
74 \\
17 \\
11 \\
5 \\
5 \\
3 \\
2\end{array}$ & $\begin{array}{l}41.9 \\
34.0 \\
20.6 \\
17.1 \\
3.9 \\
2.5 \\
1.2 \\
1.2 \\
0.7 \\
0.5\end{array}$ \\
\hline Postoperatoria tardía & $\begin{array}{l}\text { Otras } \\
\text { Incontinencia urinaria } \\
\text { Recurrencia de celes } \\
\text { Prolapso de cúpula } \\
\text { Dispareunia } \\
\text { Fístulas }\end{array}$ & $\begin{array}{r}26 \\
24 \\
12 \\
5 \\
5 \\
3\end{array}$ & $\begin{array}{l}6.0 \\
5.6 \\
2.8 \\
1.2 \\
1.2 \\
0.7\end{array}$ \\
\hline
\end{tabular}


b) Postoperatorias inmediatas: Tres muertes ocurrieron en este período. La complicación más frecuente fue la retención urinaria con 181 casos (41.9\%); hubo 147 casos $(34 \%)$ de fiebre y 89 casos $(20.6 \%)$ de infección urinaria; el absceso de cúpula se presentó en 74 casos $(17.1 \%)$.

c) Postoperatorias tardias: Se hallaron muy pocas complicaciones tardías y en muy bajo porcentaje. La más frecuente fue la incontinencia urinaria.

14. Incontinencia urinaria y grado de uretrocele: En la tabla № 9 puede observarse el predominio del uretrocele Grado II sobre los demás, y cómo la incontinencia urinaria se presenta primordialmente en este grado de uretro. cele con 114 casos $(55.1 \%)$ del total de los 207 que registra la tabla.
15. Incontinencia urinaria y grado de cistocele: En la tabla № 10 encontramos datos similares a los referentes al uretrocele, con predominio de la incontinencia urinaria en los cistoceles Grado II con 128 casos del total de 207 $(61.8 \%)$. Nótese que sólo hubo un caso $(0.5 \%)$ de incontinencia urinaria sin cistocele.

16. Retención urinaria y tipo de tratamiento: Se observa la alta incidencia de la retención urinaria o residuo alto en las cirugías para corregir los celes, que llega prácticamente al $50 \%$ de los casos intervenidos. No existe diferencia entre la colpoperinorrafia y la histerectomía vaginal (ya que en ambas se presentó en el $48 \%$ de los casos). Pero sí hay diferencia con la colporrafia anterior, en la cual se presentó en el $30 \%$ de los casos. Gráfico N 4 .

TABLA N $N 9$

Incontinencia urinaria y grado de uretrocele

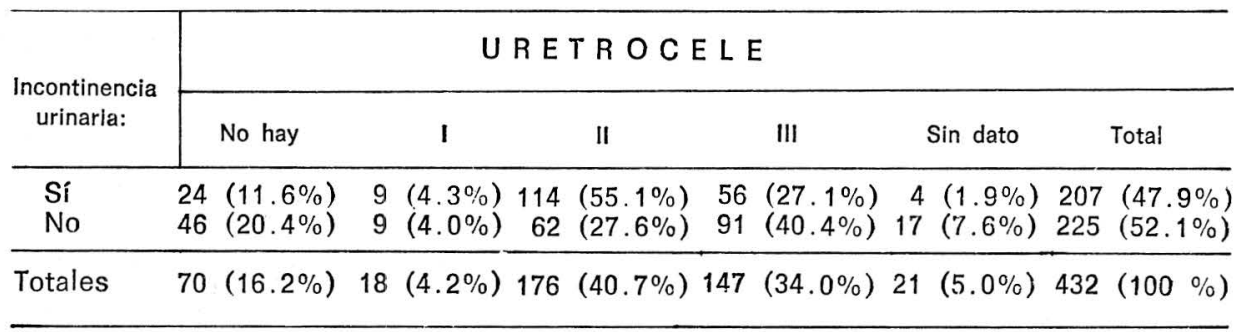

TABLA N: 10

Incontinencia urinaria y grado de cistocele

\begin{tabular}{|c|c|c|c|c|c|c|c|c|c|}
\hline \multirow{2}{*}{$\begin{array}{l}\text { Incontinencia } \\
\text { urinaria: }\end{array}$} & \multicolumn{9}{|c|}{ CISTOCELE } \\
\hline & No hay & & 1 & & II & & III & Sin dato & Total \\
\hline $\begin{array}{l}\text { Sí } \\
\text { No }\end{array}$ & $\begin{array}{r}1(0.5 \%) \\
19(8.4 \%)\end{array}$ & $\begin{array}{l}12 \\
11\end{array}$ & $\begin{array}{l}(5.8 \%) \\
(4.9 \%)\end{array}$ & $\begin{array}{r}128 \\
90\end{array}$ & $\begin{array}{l}(61.8 \%) \\
(40.0 \%)\end{array}$ & $\begin{array}{r}66 \\
103\end{array}$ & $\begin{array}{l}(31.9 \%) \\
(45.8 \%)\end{array}$ & $2 \begin{array}{l}0.0 \\
2(0.9 \%)\end{array}$ & $\begin{array}{l}207(47.9 \%) \\
225(52.1 \%)\end{array}$ \\
\hline Totales & $20(4.6 \%)$ & 23 & $(5.3 \%)$ & 218 & $(50.5 \%)$ & 169 & $(39.1 \%)$ & $2(0.5 \%)$ & $432(100 \%)$ \\
\hline
\end{tabular}


GRAFICO No. 4



17. Permanencia postoperatoria en el hospital: La gran mayoría de los casos tuvieron una permanencia postoperatoria en el hospital de 6 a 10 días, 286 casos $(66.2 \%)$. En un porcentaje bajo 50 casos: $(11.6 \%)$ la permanencia fue menor de 5 días; y en un número apreciable de casos la permanencia excedió de los 10 días (96 casos: $22.2 \%$ ). (Tabla N: 11).

18. Seguimiento: Hay un número considerable de casos que no asistieron a ningún control (105: 24.3\%), y sólo pudo llevarse control postoperatorio por más de un año en 98 casos $(22.7 \%)$. El total controlado fue de 327 casos $(75.7 \%)$ (Tabla № 12 ).

19. Medicación postoperatoria: La gran mayoría de los pacientes recibieron
TABLA N 11

Permanencia postoperatoria en el hospital

\begin{tabular}{rrr}
\hline Dias hospital & Casos & $\%$ \\
\hline $1-5$ & 50 & 11.6 \\
$6-10$ & 286 & 66.2 \\
$11-15$ & 76 & 17.6 \\
$16-20$ & 10 & 2.3 \\
Más de 20 & 10 & 2.3 \\
\hline
\end{tabular}

tratamiento con antibióticos $(70.4 \%)$ o con quimioterápicos $(27.8 \%)$ y sólo 8 pacientes $(1.9 \%)$ no recibieron ni antibióticos ni quimioterápicos. Esto significa que el $98.2 \%$ del total de 432 recibió tratamiento con quimioterápicos, con antibióticos, o en combinación ( $\mathrm{Ta}$ bla $N^{\circ}$ 13). 
TABLA N $\mathrm{N}: 12$

Seguimiento

\begin{tabular}{rrr}
\hline \multicolumn{1}{c}{ Meses } & Casos & \multicolumn{1}{c}{$\%$} \\
\hline $1-6$ & 170 & 39.4 \\
$7-12$ & 59 & 13.6 \\
$13-18$ & 32 & 7.4 \\
$19-24$ & 16 & 3.7 \\
Más de 24 & 50 & 11.6 \\
Sin control & 105 & 24.3 \\
\hline Total & 432 & 100.0 \\
\hline
\end{tabular}

TABLA № 13

Medicación postoperatoria

\begin{tabular}{lrr}
\hline \multicolumn{1}{c}{ Medicación } & № & \multicolumn{1}{c}{$\%$} \\
\hline Antibióricos & 304 & 70.4 \\
Quimioterápicos & 120 & 27.8 \\
Sin medicación & 8 & 1.9 \\
\hline Total & 432 & 100.0 \\
\hline
\end{tabular}

20. Anatomía patológica: La alteración endometrial más frecuente fue e! endometrio atrófico en 123 casos (28.5 $\%)$; luego la leiomiomatosis en 55 casos $(12.7 \%)$; metaplasia escamosa en 36 casos $(8.3 \%)$ y la hiperplasia endometrial en 25 casos $(5.8 \%)$, las cuales se dividen así: 16 adenomatosas $(64 \%), 6$ quísticas $(24 \%)$ y 3 sin determinar $(12 \%)$. Se encontraron 6 carcinomas in situ del cervix $(1.4 \%)$ y un adenocarcinoma de endometrio $(0.2 \%)$. (Tabla $\mathrm{N}^{\circ}$ 14).

\section{Comentarios}

En la tabla № 5 se observan casos en los cuales no había prolapso o este era grado I en las diferentes estructuras (columnas 1 y 2), lo que no quiere decir que tratamos pacientes sin prolap-
TABLA N $: 14$

Anatomía patológica

\begin{tabular}{lrr}
\hline \multicolumn{1}{c}{ Anatomía patológica } & Casos & \multicolumn{1}{c}{$\%$} \\
\hline Endometrio proliferativo & 130 & 30.1 \\
Endometrio atrófico & 123 & 28.5 \\
Endometrio secretor & 83 & 19.2 \\
Leiomiomatosis & 55 & 12.7 \\
Metaplasia escamosa & 36 & 8.3 \\
Hiperplasia endometrial & 25 & 5.8 \\
Displasia cervical & 11 & 2.5 \\
Can in situ & 6 & 1.4 \\
Ca endometrio & 1 & 0.2
\end{tabular}

so o con prolapso grado I sino que estas estructuras no prolapsadas estaban asociadas a descensos II o III en cualquiera de las otras estructuras aquí consideradas.

El desgarro perineal se encontró asociado al prolapso genital en el $69.6 \%$ de los casos pero notando que existe un gran número de casos $112(26 \%)$ en los cuales existiendo rectocele no se mencionó el grado de desgarro perineal. En el análisis de la edad y el grado de histerocele se encuentran los siguientes hechos:

1. El grado de prolapso tiene tendencia a aumentar con la edad de la paciente, esto posiblemente debido a la deficiencia estrogénica que se presen. ta después de la menopausia que se manifiesta clínicamente por disminución considerable del trofismo de los órganos genitales.

2. No pudimos confirmar el aumento de la frecuencia de complicaciones urinarias como infección o hidronefrosis en los prolapsos uterinos grado III, pero la mayoría de los autores han confirmado este hallazgo que hace obligatorio el tratamiento quirúrgico del prolapso, antes de que este llegue a ser grado III 11. 
Hoy el manejo quirúrgico del prolapso de la cúpula en el Hospital San Ignacio cuando la cúpula lo permite, es la fijación presacra combinada con uretrocistopecia tipo Marshall y no la operación de Fletcher, que fue lo encontrado en este estudio.

El tratamiento del histerocele la cirugía de elección sigue siendo la histerectomía vaginal, dejando la operación de Mánchester para las pacientes que no desean perder su función reproductiva y la operación de Le Fort para aquellas pacientes postmenopáusicas con riesgo quirúrgico muy alto y sin actividad sexual.

En las complicaciones notamos un porcentaje elevado de transfusiones 238 casos $(55.1 \%)$, pero de las cuales 128 requirieron sólo $500 \mathrm{cc}$ de sangre lo cual cuestiona un poco la verdadera necesidad de transfundir $\ni$ estas pacientes y nos hace pensar si no sería un poco de precipitación por parte de los anestesióloaos.

La complicación postoperatoria inmediata más frecuente fue la retención uri. naria, sin embargo para algunos autores este es un dato de buen pronóstico para aquellas pacientes que presentaban asociada al prolanso la IUE 16 .

Es importante comentar que la permanencia promedio postoperatoria tan elevada, se debió a que la salida de las pacientes estaba sujeta a la micción espontánea o al residuo postmiccional menor de 100 cc. Esta investigación permitió que dicha conducta se modificara sustancialmente ya que hoy toda paciente sale al $5^{\circ}$ día postoperatorio, haya o no presentado retención urinaria. Si presenta retención se dará salida con sonda y controles posteriores con el fin de medir residuo vesical postmiccional.

El uso de antibiótico o quimioterápi$\cos$ no los consideramos profilácticos sino terapéuticos ya que estas pacientes requieren, en su mayoria, sonda vesical permanente de 5 días y sin posibilidad de usar bolsas asépticas por falta de recursos económicos. Por otra parte, consideramos que el campo vaginal es siempre en mayor o menor grado un campo séptico.

\section{Resumen}

Se revisaron todas las historias de pacientes intervenidas para corrección del prolapso genital en un período de 6 años comprendido entre el $1^{\circ}$ de enero de 1966 y el 31 de diciembre de 1971.

Se reunieron 432 historias que constituyen el universo de esta investigación.

Los datos se recoletaron con un tormulario precodificado y el procesamiento de los datos por computador.

\section{Resultados}

1. El prolapso se presenta principalmente entre los 40 y 59 años, siendo más frecuente en grandes multíparas.

2. La asociación con carcinoma in situ es rara y no se encontró ningún caso de carcinoma invasor.

3. La patología asociada más frecuente fue la IUE.

4. La presencia del desgarro perineal no fue detectada en forma cuidado. sa en el examen clínico preoperatorio.

5. El grado de prolapso tiene tendensia a aumentar proporcionalmente con la edad, pero esta relación no es igualmente proporcional con el aumento de la paridad.

6. El tipo de histerectomía no incidió en la aparición o no del prolapso de cúpula. 
7. El principal tratamiento utilizado para la corrección del prolapso genital fue la histerectomía vaginal, dejando los tratamientos conservadores principalmente para las menores de 30 años.

8. La complicación operatoria más frecuente fue el sangrado abundante y las complicaciones postoperatorias inmediatas más frecuentes fueron: la retención urinaria, la fiebre, la infección urinaria y el absceso de cúpula. Las 3 muertes correspondientes a 2 sepsis y una a shock hemorrágico.

9. La IUE es más frecuente en los prolapsos grado II.

10. La retención urinaria no tuvo diferencia significativa, entre la colpoperineorrafia y la histerectomía vaginal, con $\mathrm{GC}$.

11. La permanencia postoperatoria en el hospital mayor de 5 días fue muy alta, $(88.4 \%)$ de los casos.

12. El seguimiento postoperatorio fue pobre y sólo se pudo hacer un seguimiento de más de un año en 98 casos $(22.7 \%)$.

13. En la mayoría de los casos se utilizó medicación postoperatoria con antibióticos o quimioterápicos $(98.2 \%)$.

\section{Conclusiones}

1. El prolapso genital es más frecuente en la edad media de la mujer y en las grandes multíparas

2. El grado de prolapso aumentó proporcionalmente con la edad, pero no con la paridad.

3. El tratamiento quirúrgico de elección en el histerocele es la histe. rectomía vaginal.
4. La cirugía vaginal tiene tendencia al sangrado abundante.

5. La retención urinaria es la complicación postoperatoria inmediata más frecuente, pero esto no debe aumentar la permanencia hospitalaria.

6. El seguimiento fue pobre, lo que es un común denominador en nuestras pacientes.

7. El uso de antibióticos o quimioterápicos en la cirugía vaginal no es profiláctico sino terapéutico.

8. La patología asociada más frecuente fue la IUE y principalmente en los uretrocistoceles grado II.

\section{GENITAL PROLAPSE TREATMENT}

\section{Conclusions}

1. Genital prolapse is more frequent in middle age and multiparous women.

2. The degree of prolapse increased proportionally with age, but not with parturition.

3. The eligible surgical treatmen in the hysterocele is vaginal hysterectomy.

4. Vaginal surgery tends to produce great bleeding.

5. Urinary retention is the most frequent immediate postoperative complication; however, this problem must not increase the staying at the hospital.

6. The follow-up was poor, which represents a common denominator in our patients.

7. The use of antibiotics or chemotherapy products in vaginal surgery is not prophylactic but therapeutical. 
8. The most frequent associated pathology was IUE, and mainly in grade II urethrocystoceles.

\section{Bibliografía}

1. GAVIRIA S., Dagoberto y VELEZ Z., Antonio. "Prolapso Genital". Tratamiento Quirúrgico. (Revista Colombiana de Obstetricia y Ginecología. Vol. XVI. No 2, p. 145, 1966).

2. MILANES J., Jorge. "Perineorrafia Modificada". (Revista Colombiana de Obstetricia y Ginecología. Vol XVII. N², p. 165, 1966).

3. MARIN A., Laureano. "Colpocleisis de Le Fort". (Revista Colombiana de Obstetricia y Ginecología. Vol. XVIII. № 6, p. 415 , 1967).

4. GOMEZ G., Rafael. "Postoperatorio en cirugía vaginal". (Revista Colombiana de Obstetricia y Ginecología. Vol. XVI. N: 3, p. 179, 1965).

5. ALBA P., Luis. "Cuidados postoperatorios en cirugía vaginal". (Revista Colombiana de Obstetricia y Ginecología. Vol. XVI. No 3, p. 259, 1965).

6. FONNEGRA M., Alvaro y GUZMAN A. Rubén Darío. "Tratamiento del prolapso de cúpula vaginal por la colpocistopexia vía abdominal". (Revista Colombiana de Obstetricia y Ginecología No 6, p. 631, 1963).

7. CACERES A., Eduardo y TURBAY B., Salomón. "Tratamiento de la cúpula en la histerectomía vaginal". (Revista Colombiana de Obstetricia y Ginecología. Vol. XIV. No 6. 1963).
8. DIAZ G., Jaime. "Corrección del prolapso de cúpula vaginal mediante la sacrocolpopexia. (Revista Colombiana de Obstetricia y Ginecología". Vol. XXI. № 1, p. 39, 1971).

9. EMGE A., Ludwig y DURFEE, R. B. "Pelvic Organ Prolapse four thousand years of treatment". Clinical Obstetrics and Gynecology. Vol. 9. N 4, p. 993, December, 1966.

10. THOMPSON D., John y LYON B., James. "Vaginal Hysterectomy". Clinical Obstetrics and Gynecology. Vol. 9. No 4, p. 1.033. 1966.

11. RICHARD W., Telinde. "Prolapse of the Literus and Alliend Conditions". American Journal of Obstetrics and Gynecology. Vol. 94, p. 444, 1966.

12. WAYNE F., Banden. THOMAS A., Walker "Statical Evaluation of Vaginal Relaxation". Clinical Obstetrics and Gynecology. Vol. 15, N: 4, December, 1972.

13. PARSONS Y SOMMERS. "Gynecology $W$. B. Saunders Company", p. 1.009.

14. MEDINA MURILLO, Jorge E. DE MESA, Lucely. "Incontinencia urinaria de esfuerzo en la mujer. Estudio radiológico". (Revista Colombiana de Obstetricia y Ginecología. Vol. XXI. No 3, mayo-junio, 1970).

15. DIAZ GARZON, Jaime. "Corrección del prolapso de cúpula vaginal mediante la sacrocolpopexia". (Revista Colombiana de Obstetricia y Ginecología". Vol 22. N: 1, 1971).

16. ANGULO ROA, Alberto y URIZA GUTIERREZ, Germán. "Valor del uretrocistograma con cadena en el tratamiento de la incontinencia urinaria de esfuerzo. (Revista Colombiana de Obstetricia y Ginecología". Vol. XXXI, $\mathrm{N}^{\circ} 2,1980$ ). 